\title{
From a Personal Learning Environment to an Adaptable Personal Learning Environment: meeting the needs and preferences of disabled learners
}

\author{
Elaine Pearson, Voula Gkatzidou, Steve Green \\ Accessibility Research Centre, Teesside University, \\ United Kingdom \\ Email: e.pearson@tees.ac.uk
}

\begin{abstract}
Researchers and educational technologists are striving to achieve e-learning solutions that offer choice and flexibility in the time, place and mode of learning. Developments that centre on user preference and personalisation have yet to make a significant impact on institutional learning environments and this limitation has given rise to the concept of the Personal Learning Environment. This paper considers a system that takes this concept one step further to cater for the needs and preferences of disabled learners - an Adaptable Personal Learning Environment.
\end{abstract}

Keywords: accessibility, adaptability, Personal Learning Environment, standards, metadata

\section{Introduction}

The 'traditional' Learning Management System (LMS) has, over the past ten years, become an accepted part of e-learning provision. For disabled students in particular, the LMS has increased opportunities to participate in mainstream education that were previously much more difficult or impossible [7]. However, these systems are limited in their support for personal choice, flexibility and pedagogical freedom. A Personal Learning Environment [5] is one that replaces some or all of the features of a standard LMS with tools that are personal to the learner and integrated with the student's own personal system. The interface and tools are the personal choice of the learner rather than prescribed by the institutional LMS. An Adaptable Personal Learning Environment (APLE) takes the concept of adaptability as defined by the IMS AccessForAll [4] to create an accessible, personalised and flexible learning environment, aimed particularly, but not exclusively, at disabled students.

\section{Characteristics of an APLE}

Adaptability is based on the recognition that people with sensory, cognitive or motor impairments may have needs that make access not just inconvenient but impossible if not met. In previous papers we have reported on those completed aspects of our work that individually form components of an APLE, e.g. [1],[2]. From this we are able to define the characteristics of an
APLE. It should include a framework that supports alternative interfaces, applications, learning objects and interactions; a means for learners to establish a profile of their needs and preferences for access and interaction; a means of creating adaptable learning objects by teachers with limited development skills; a method for supporting the adaptation of learning objects according to the learners profile; a community of practice to share, propose, define or develop aspects of the APLE; and support for standards to facilitate interoperability. This paper briefly discusses the current components of the APLE, followed by a consideration of further work, specifically standards for interoperability between learning environment, devices and learning objects and the development of a community of practice.

\section{Portland Personal Learning Environment}

A previous research project involved the development of an adaptable, symbols based PLE for students with severe disabilities. The project involved Portland a UK specialist college, partners from tertiary education, and the private sector. The Portland user group is young adult learners aged 16 years upwards with profound and multiple disabilities. The Portland PLE [3] interface, learning resources and interactions were derived from learner profiles established through observations and formal assessment. It includes the standard features found in most mainstream LMS's, but is designed specifically to meet the individual needs and preferences of this target user group. The interface is personalised to allow learners to have the screen display and layout of their choice, and choice of symbol set (PCS, Rebus or Makaton). It meets the needs of those learners with low literacy levels through symbol-supported text and speech output. Interaction is tailored to students' preferred input device (e.g. mouse, switch, scanning). Portland's unique functionality and features make it an accessible and adaptable environment for learners with severe learning difficulties and physical disabilities. The PLE is in use by the students and tutors who were involved in its development. An Adaptable Personal Learning Environment (APLE) would extend these aspects of 
adaptation further to meet the needs of any learner. This requires a common set of standards to be identified for interoperability to enable personalisation from available components.

\section{Profile for Adaptable Learning Tool}

In the Portland PLE, learners profiles were submitted direct to the system by the tutors. In our APLE the learners' characteristics are expressed, stored, retrieved and altered through the Profile for Adaptable Learning (PAL) tool. It implements the IMS ACCLIP specification [4] to provide an easy way for the learner to create a profile that conforms to that specification. ACCLIP describes the user in terms of accessibility needs by using a XML-based syntax. It enables the description of user preferences (visual, aural or device), which can be usefully exploited for tailoring learning content (e.g. preferred/required input/output devices or preferred content alternatives). The profile is anonymous, in that there is no need to know who the user is, or why they require the specified support. Choices and options can be expressed as well as absolute needs; for example a user might express a preference for Braille output, but with an indicator that auditory substitution is also acceptable.

Using PAL the learner can create, edit and modify their preferences for appearance, layout and selection of content. A simple wizard-style interface presents a choice of six adaptability statements, then further options refined according to their selection [1]. Learner profile information can express accessibility needs but often context or preference may be equally important e.g., a non-auditory profile might mean that audio material needs a transcription and video - captions or subtitles. In broad terms there is a need to be able to define a user's contextual profile as a set of requirements for services and resources. The profile defines the user's human-computer interaction (HCI) requirements in terms of visual, auditory and tactile components. The three main elements of the profile are: display or output (typically visual but could be auditory or tactile); control or input (typically keyboard and mouse but could be switches, touch-screen, tactile devices or voice recognition); content (primarily visual, auditory media or textual components which can be read or transformed into auditory components by a screen reader). Accessibility, therefore, is the ability of the environment to adjust to the needs of all learners and is determined by the flexibility of the environment (with respect to presentation, control methods and access modality) and the availability of adequate alternative-but-equivalent content. Declared needs and preferences may change according to context [5].

\section{A Learning Object Authoring Tool}

The APLE should provide a straightforward and intuitive way to create accessible and adaptable learning objects. The Learning Object Tutor Tools Interface (LOTTI) supports the production of retrievable, re-usable and adaptable learning objects [1]. Using a two stage design process, the general pedagogical structure of the object is developed then specific alternative learning content is identified and added to a template. The authoring process is based on a library of pedagogical design patterns. Once a template has been selected, authors can add different subject specific content to produce a learning object.

LOTTI adopts the IEEE Learning Object Metadata specification to describe information on the component structure of the learning object; and the IMS accessibility metadata specification (AccMD) to describe the accessibility of a learning resource by specifying the content and its available equivalent or alternative form. The finished learning objects are packaged in accordance with the IMS Content Packaging specification to facilitate export to a learning repository.

\section{The Transformation Augmentation and Substitution Service}

To achieve an accessible relationship between a resource and the user, descriptions of user needs and preferences must be checked against descriptions of resource components until they match. This process involves a description of a user's control, display and content needs and preferences being matched with a description of the components of the learning object to form an accessible relationship between the user and the learning object. According to the AccessForAll metadata overview, accessible systems should be able to adjust the user interface, locate needed resources and alter resource properties to match the needs and preferences of the user. We have developed a transformation, augmentation and substitution service (TAS) that represents an instance of an AccessForAll service. Transformation could be text rendered visually, or aurally, or in a tactile form as Braille. Augmentation involves the optional addition of a feature to a primary resource, e.g. a textual caption could be added to a video when required by a user with a hearing impairment or in a noisy environment. 
Substitution could be the replacement of a visual element with components that match the user's preferences of vision-free access. For an APLE, this service must be applied not only to learning objects but also to interfaces, services and components.

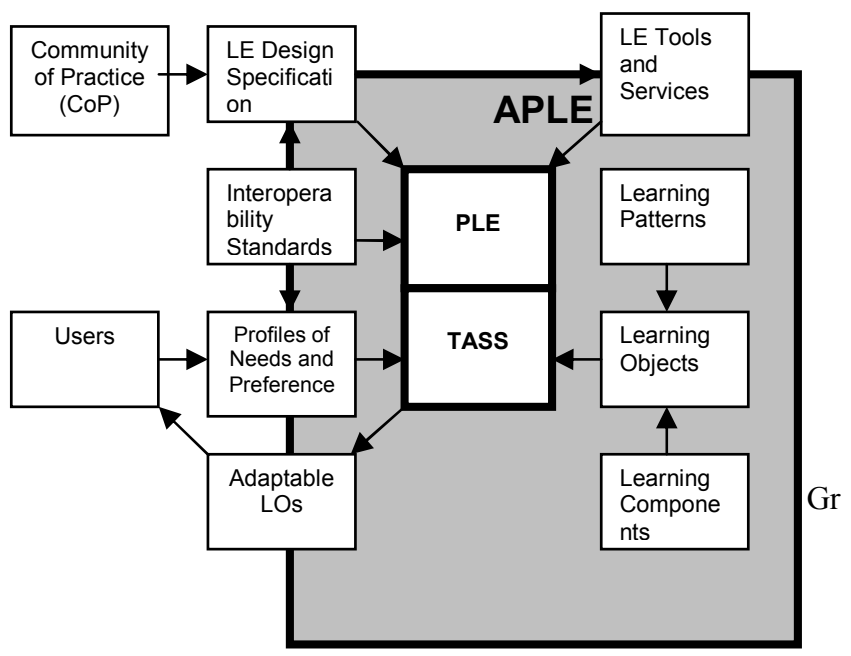

Figure One: An Adaptable Personal Learning Environment

\section{Further work}

To date several essential components of the APLE have been developed - the profile tool (PAL) to enable learners to specify their needs and preferences; a learning object authoring tool (LOTTI) that supports the design and development of accessible learning objects and their alternatives, and a transformation, augmentation and substitution service (TAS) to effect the matching of accessible components and learners profiles. The experience gleaned from the Portland PLE project provides the basis for a framework to support a fully Adaptable Personal Learning Environment.

The APLE must enshrine common models to facilitate adoption of available standards. Currently they include the IMS AccessForAll specifications for learning objects and learner profiles, and Dublin Core and SCORM standards for e-learning. While the complexity of systems make the negotiation of a single set of technical standards a practical impossibility [15], we can at least identify those existing standards and map the requirements for interoperability. Thus, gaps and inconsistencies can be established and strategies developed to consider how they can be addressed. An APLE that aims to meet the needs of a wide range of learners, often with very specific requirements cannot be achieved by any one group of researchers or developers so a Community of Practice comprising of researchers, developers, learners, educational technologists, tutors, students, standards experts and assistive technologists is essential. There are already examples of COPs producing accessible open source solutions (e.g. AccessApps an initiative supported by the JISC Regional Support Centres (RSC) and JiscTechDis) [8]. A collaborative approach to elearning development will ensure an adaptable model that will grow and change according to the needs of the user.

\section{References}

[1] Gkatzidou, S. \& Pearson, E. (2009). A transformation, augmentation, substitution service (TASS) to meet the needs and preferences of the individual learner. Paper presented at the IEEE International Conference of Advanced Learning Technologies (ICALT). Riga, Latvia.

[2] Green, S., Jones, R., Pearson, E., \& Gkatzidou, S. (2006).Accessibility and adaptability of learning objects: responding to metadata, learning patterns and profiles of needs and preferences. ALT-J Research in Learning Technology, 14(1), pp. 117-129.

[3] Harrison, M Stockton C., \&Pearson, E. (2008). Inclusive, Adaptive Design for Students with Severe Learning Disabilities. The 8th IEEE International Conference on Advanced Learning Technologies, Santander, Cantabria, Spain

[4] IMS. (2004). IMS Global learning/Dublin Core AccessForAll project. Available online: http://www.imsglobal.org/accessibility Retrieved 20/01/09

[5] JISC (2005). Personal Learning Environments. Available online: http://www.jisc.ac.uk/index.cfm?name=cetis ple Retrieved 20/01/09

[6] Nevile, L., Cooper, M., \& Heath, A. (2005). Learnercentred Accessibility for Interoperable Web-based Educational Systems. 14th International World Wide Web Conference, Chiba, Japan. Available online http://13s.de/ olmedilla/events/interopPapers/paper08.pdf Retrieved 20/01/09

[7] Pearson, E., \& Koppi, T. (2002). Inclusion and online learning opportunities: designing for accessibility, Association for Learning Technology Journal, 10(2).

[8] JISC, RSC, Scotland, (2010),AccessApps, http://www.rsc-scotland.ac.uk/eduapps/accessapps.php, accessed 25.01.10 